\title{
Multidimensional scaling of D15 caps: Color-vision defects among tobacco smokers?
}

\author{
DAVID BIMLER AND JOHN KIRKLAND \\ Department of Health and Human Development, Massey University, New Zealand \\ (ReCEIVED September 7, 2003; ACCEPTED January 8, 2004)
}

\begin{abstract}
Tobacco smoke contains a range of toxins including carbon monoxide and cyanide. With specialized cells and high metabolic demands, the optic nerve and retina are vulnerable to toxic exposure. We examined the possible effects of smoking on color vision: specifically, whether smokers perceive a different pattern of suprathreshold color dissimilarities from nonsmokers. It is already known that smokers differ in threshold color discrimination, with elevated scores on the Roth 28-Hue Desaturated panel test. Groups of smokers and nonsmokers, matched for sex and age, followed a triadic procedure to compare dissimilarities among 32 pigmented stimuli (the caps of the saturated and desaturated versions of the D15 panel test). Multidimensional scaling was applied to quantify individual variations in the salience of the axes of color space. Despite the briefness, simplicity, and "low-tech" nature of the procedure, subtle but statistically significant differences did emerge: on average the smoking group were significantly less sensitive to red-green differences. This is consistent with some form of injury to the optic nerve.
\end{abstract}

Keywords: Multidimensional scaling, Color-vision deficiency, D15 panel test, Smoking

\section{Introduction}

Tobacco consumption, on its own or aggravated by alcohol and poor nutrition, can affect color vision. Even smokers with no overt ophthalmological disease, compared to age-matched nonsmoking controls, evinced elevated scores on a sensitive test of color discrimination - the Roth 28-Hue Desaturated panel test (Erb et al., 1999). The effect was dose dependent.

This is reasonable, given the vulnerability of the optic nerve and retina to insults such as anoxia or toxic exposure: they are part of the central nervous system, with highly specialized cells and high metabolic demands. Tobacco smoke contains a range of compounds including nicotine, cyanide, and carbon monoxide (CO). Inhaling low levels of CO increases dark-adaptation time and the dark-adapted threshold of light detection; moreover, these changes are greater among smokers (von Restorff \& Hebisch, 1988). Cigarette smoking is perhaps the commonest form of toxic exposure in many Western countries.

Polarity of visual deterioration provides a clue as to the locus of the damage. According to Köllner's law, acquired red-green deficits (in which colors along a red-green axis of color space become harder to discriminate, while differences along that axis contribute less to perceived color dissimilarities) are associated

Address correspondence and reprint requests to: David Bimler, 14 Akatea Street, Berhampore, Wellington, New Zealand. E-mail: d.bimler@massey.ac.nz with insults to the optic nerve as opposed to the retina. Fletcher and Voke (1985, Tables 4.2 and 4.5) list tobacco and CO as potential causes of red-green disturbances. Tobacco exposure can cause toxic optic neuropathy (TXON), that is, damage to the optic nerve (Krastel \& Moreland, 1991). Symptoms include color-vision deficiency, especially red-green deficits. However, the Roth 28-Hue errors in Erb et al. (1999) were not concentrated along any specific direction in color space, and provided no evidence for a polar deficit in the smoking groups-in contrast to the usual situation with TXON.

Here we examine color vision in smokers, working within a long-standing research tradition in which the variations among observers are probed by asking them to assess the dissimilarities they perceive among color stimuli (Helm, 1964; Chang \& Carroll, 1980; Offenbach, 1980; Shepard \& Cooper, 1992). Research in this tradition often involves analyzing the dissimilarity judgements with multidimensional scaling (MDS). The special case of individual-differences MDS algorithms can render the data down to a small number of parameters for each observer, specifying how that observer's perceptual color space departs from the consensus (Chang \& Carroll, 1980). Color-vision deficiencies can be considered in this way as personal distortions of a consensual space, making them amenable to quantification with MDS (Farnsworth, 1943; Shepard \& Cooper, 1992; Bimler et al., 2000). For instance, any reduction in the salience of a red-green axis manifests as a specific pattern of discrimination and dissimilarity judgements, and in geometrical terms can be quantified as a specific compression of color space along that axis. 
If two stimuli are indiscriminable (treated as interchangeable), the points representing them in the observer's color space cannot be far apart. Thus, discrimination data (thresholds) focus on local properties of personal color spaces. In contrast, dissimilarity data (suprathreshold) are potentially more sensitive to polar deficits because they focus on a global property or distortion, distributed across large as well as small distances in color space.

In the present study, observers were presented with a series of triads of color stimuli (pigment swatches), and asked which stimulus pair (of the three pairs in each triad) was the most similar, that is, more similar than the other two. For MDS analysis, these "triadic data" were treated as ordinal dissimilarity comparisons.

The present stimuli have previously been represented as a configuration of points within color space (written as $\mathbf{X}_{0}$ ), by MDS analysis of separate data (Bimler et al., 2004). Analysis here consisted of compressing and elongating $\mathbf{X}_{0}$ optimally along its axes [red-green ( $\mathrm{R}-\mathrm{G})$, blue-yellow (B-Y), lightness] to produce for each subject a configuration $\mathbf{X}_{m}$ that maximized the likelihood of generating his or her judgements (Bimler et al., 2000).

\section{Materials and methods}

Forty-three nonsmokers (20 male \& 23 female) and 41 smokers (29 male \& 12 female) were recruited from students and staff at the universities of Massey (NZ) and Oakland (MI, US). Median ages were 20 and 22 years, respectively, with most ages in the range 18-34 years, and no gender difference. "Smokers" had been smoking at least a packet of cigarettes per day, for at least a year. All subjects were screened for color-vision deficiency using the D15 and D15-DS panel tests. They were asked about any eye problems, and whether color-vision deficits or other ophthalmological conditions had been diagnosed in the past.

Stimuli were the 32 caps of the D15 and D15-DS color-vision panel tests $(1.2 \mathrm{~cm}$ circles of pigment-coated paper in black plastic mounts, subtending an angle of $1.5 \mathrm{deg}$ at normal viewing distance). In Munsell notation, the 16 caps of the D15 have Value 5 and Chroma 4, with the exception of one cap with Chroma $=6$ (Fletcher \& Voke, 1985). The D15-DS caps are lighter (Value 8) and less saturated (Chroma 2). The same sets of caps were used to collect data at both locations.

They were viewed resting on a sheet of gray card (N5), within a desktop booth of walls and baseboard painted matt gray. Two Philips 'TL'D /950 fluorescent tubes (color temperature $5300 \mathrm{~K}$, Color Rendering Index > 95), suspended by light cord from struts extending above the booth's walls, provided a luminance level that was measured by photometer and held constant at 500 lux by raising or lowering the two tubes (Bimler et al., 2004).

Stimuli were shuffled into five random triads with one left over at random each time. Subjects indicated the most-similar pair in each triad, by choosing the "odd-one-out" stimulus (most dissimilar). This procedure was performed with the D15 and D15-DS sets separately; then repeated 12 times with a mixed set (in which caps $0,3,6,9,12$, and 15 in the D15 sequence were replaced by their desaturated counterparts). In total, each subject made 70 odd-oneout decisions. No time limits were set on the responses. The entire process took 15-20 min. To protect the stimuli and avoid contrasting color stimulation, subject and researcher wore white gloves while making and recording these judgments.

\section{Analysis}

The multidimensional-scaling paradigm attempts to account for the data by representing the stimuli as points in a geometrical model, arranged so that the distance between each pair of points reflects the dissimilarity between the corresponding stimuli. We create a separate solution $\left(\mathbf{X}_{m}\right)$ for each observer $m . \mathbf{X}_{m}$ is a matrix of coordinates, in which the $i$ th row locates the $i$ th point: $\mathbf{x}_{m i}=$ $\left(x_{m i 1}, x_{m i 2}, x_{m i 3}\right)$. Three dimensions are required since the stimuli vary in lightness (Value) as well as along the R-G and B-Y axes of color space.

There are too few triad judgements from each observer to derive $\mathbf{X}_{m}$ from scratch. Instead we calculate weights or salience parameters for each observer, $\mathbf{w}_{m}=\left(w_{m 1}, w_{m 2}, w_{m 3}\right)$. These generate $\mathbf{X}_{m}$ by compressing or elongating a consensus solution $\mathbf{X}_{0}$ along its axes: if $\mathbf{x}_{i}$ is the $i$ th row of $\mathbf{X}_{0}$, then $\mathbf{x}_{m i}=\left(w_{m 1} x_{i 1}\right.$, $\left.w_{m 2} x_{i 2}, w_{m 3} x_{i 3}\right)$. For instance, $w_{m 1}$ is the salience of dimension 1 to the $m$ th observer. In the limiting case of zero salience, displacements along the dimension contribute nothing to color dissimilarity, and the observer perceives no difference between stimuli on one side of $\mathbf{X}_{0}$ and their counterparts on the other (for instance, points $10 \mathrm{G}$ and $2.5 \mathrm{R}$ in Fig. 1 , or $5 \mathrm{P}$ and $10 \mathrm{~B}$ ). This would be represented by compressing $\mathbf{X}_{m}$ axially until the points representing the indistinguishable stimuli coincide: $w_{m 1}=0$. This is the "weighted Euclidean" model of individual-difference MDS.

The parameters $\mathbf{w}_{m}$ were calculated by a process of iterative adjustment, until the $\mathbf{X}_{m}$ they produced had the maximum likelihood of giving rise to the actual judgements made by observer $m$. The likelihood of the personalized color space $\mathbf{X}_{m}$ giving rise to a given odd-one-out judgement is enhanced if the corresponding points form an elongated triangle, with the odd-one-out stimulus at its acute corner (for details of this Maximum Likelihood model, see Bimler et al., 2000). The likelihood that the $m$ th observer's responses would arise from $\mathbf{X}_{m}$ (averaged over all 70 triad decisions) is $l_{m}$. It ranges from 0.5 (no better than chance) up to 1 .

Naturally triangles in $\mathbf{X}_{m}$ are differently elongated compared to their counterparts in $\mathbf{X}_{0}$; perhaps in different directions. For instance, in the case where $w_{m 1}=0$ and two points coincide, the third stimulus in a triad involving that pair of stimuli will be the odd-one-out.

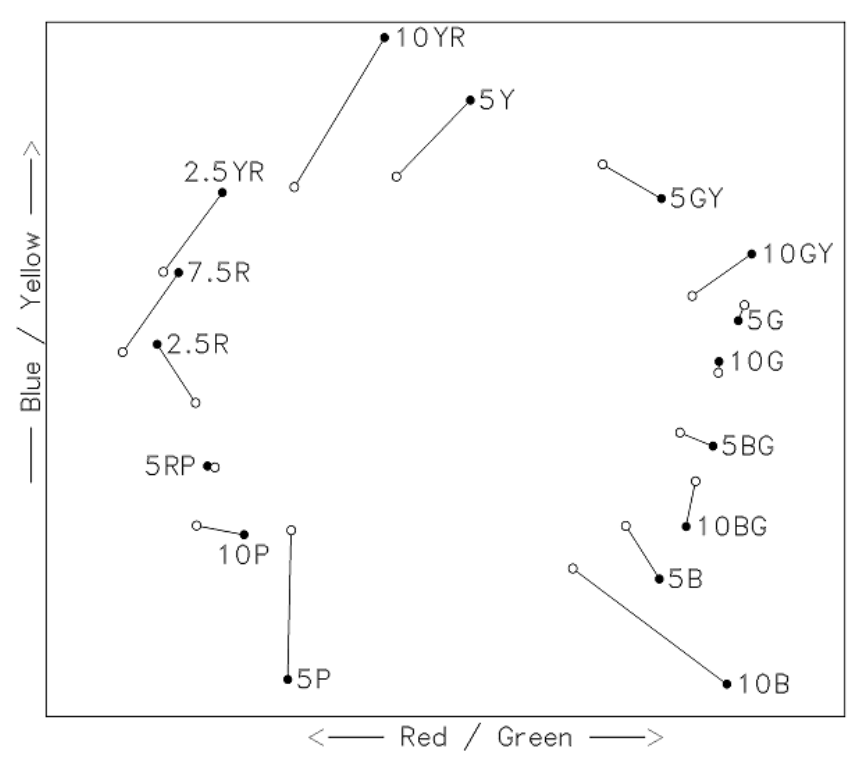

Fig. 1. First two dimensions of three-dimensional MDS solution $\mathbf{X}_{0}$. Two stimuli (linked with lines) share each Hue: a darker saturated stimulus set with Chroma $=4$, Value $=5$ (solid circles), and a lighter desaturated set with Chroma $=2$, Value $=8$ (open circles). 


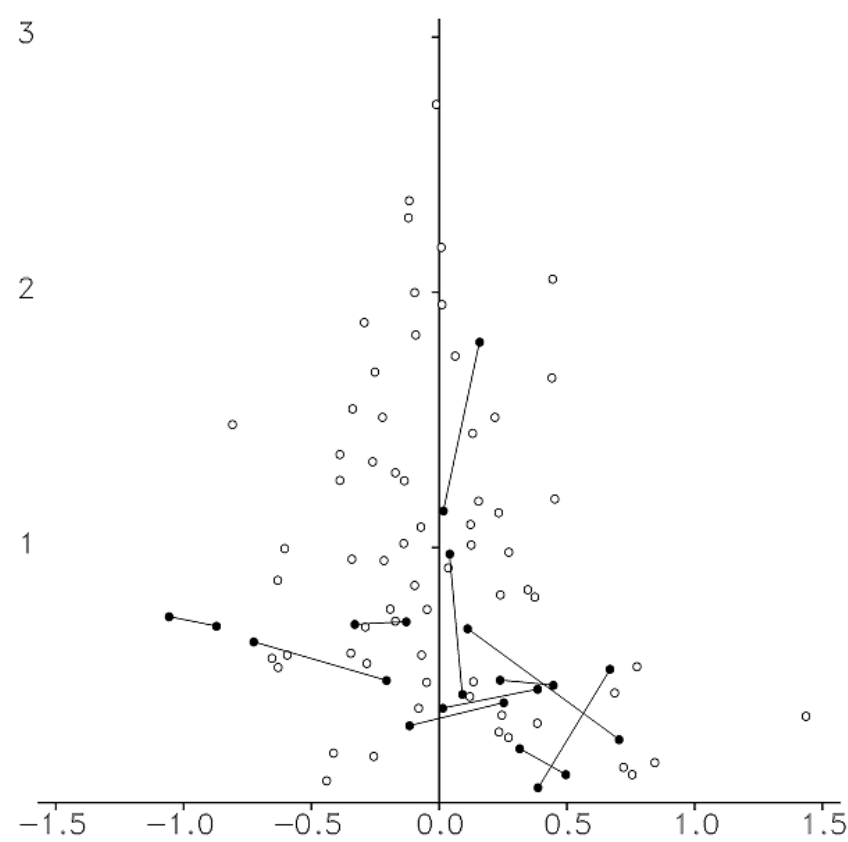

Fig. 2. Individual color-axis weighting parameters. Solid circles linked by lines represent results for individuals tested twice. Vertical scale: $w_{m 3}^{2}$ (weight of lightness axis). Horizontal scale: $w_{m 1}^{2}-w_{m 2}^{2}$ (balance between $\mathrm{R}-\mathrm{G}$ and $\mathrm{B}-\mathrm{Y}$ weights).

There is a constraint that $w_{m 1}^{2}+w_{m 2}^{2}+w_{m 3}^{2}=3$, to ensure that the sum-of-squared distances from the origin to the $\mathbf{x}_{m i}$ is the same in each $\mathbf{X}_{m}$, that is, that scale is constant across $m$.

The consensus map $\mathbf{X}_{0}$ is shown as Fig. 1. It was found by an iterative hill-climbing algorithm, similar to the MAXSCAL pro- gram for dissimilarity comparisons (Takane, 1978). The points $\mathbf{x}_{i}$ are confined to two parallel planes in space: the lighter (also desaturated) stimuli from the D15-DS are constrained to share a single value of the third dimension, while the darker (saturated) D15 stimuli share a second value (Bimler et al., 2004).

Results

Eleven subjects were tested twice. Their parameters (plotted in Fig. 2) were reasonably stable. Group mean values of $w_{m 1}^{2}, w_{m 2}^{2}$, $w_{m 3}^{2}$, and $l_{m}^{2}$ were compared: that is, sensitivity to R-G, B-Y, and lightness color differences, and overall color performance $\left(l_{m}\right.$ was squared to produce a normal distribution). Female smokers and all males showed significantly lower $w_{m 1}^{2}$ and higher $w_{m 3}^{2}$ than female nonsmokers (Tables 1a \& 1b).

Group differences in $w_{m 2}^{2}$ were small and not significant (Table 1d).

The higher $w_{m 3}^{2}$ does not necessarily mean that lightness is more salient for female smokers in absolute terms; only that lightness remains as a potential distinguishing feature on which to base an odd-one-out decision despite decreased sensitivity to differences in the color plane (defined by $\mathrm{R}-\mathrm{G}$ and $\mathrm{B}-\mathrm{Y}$ axes). The interdependence of salience parameters links a reduction in $w_{m 1}$ with increases in the other two: $w_{m 1}^{2}$ and $w_{m 3}^{2}$ were correlated $(r=$ $0.86, P<0.001)$.

Males did not show any smoking-dependent differences in color-axis sensitivity, superimposed on their group difference from females. Smoking males were lower on $l_{m}^{2}$ than nonsmoking males and all females, though not to a $P<5 \%$ level of significance (Table 1c).

\section{Discussion}

A panel test (the Roth 28-Hue Desaturated) revealed reduced color discrimination among smokers (Erb et al., 1999). That is, the

Table 1. Group means

\begin{tabular}{|c|c|c|c|c|c|c|c|}
\hline \multicolumn{4}{|c|}{ (a) $w_{m 1}^{2}$} & \multicolumn{4}{|c|}{ (b) $w_{m 3}^{2}$} \\
\hline Gender & Nonsmokers & & Smokers & Gender & Nonsmokers & & Smokers \\
\hline M & $\begin{array}{c}0.96 \pm 0.32 \\
\uparrow \\
\uparrow=0.003\end{array}$ & $P=0.003$ & $\begin{array}{c}0.96 \pm 0.39 \\
(n=29)\end{array}$ & M & $\begin{array}{l}0.93 \pm 0.55 \\
\uparrow P=0.04\end{array}$ & $P=0.01$ & $1.01 \pm 0.65$ \\
\hline $\mathrm{F}$ & $\begin{array}{r}1.32 \pm 0.42 \\
(n=23)\end{array}$ & $\stackrel{\leftrightarrow}{ }=0.02$ & $\begin{array}{l}0.97 \pm 0.39 \\
(n=12)\end{array}$ & $\mathrm{F}$ & $0.60 \pm 0.46$ & $P=0.02$ & $1.07 \pm 0.70$ \\
\hline
\end{tabular}

(c) $l_{m}^{2}$

\begin{tabular}{|c|c|c|c|c|c|}
\hline Gender & Nonsmokers & Smokers & Gender & Nonsmokers & Smokers \\
\hline $\mathrm{M}$ & $0.74 \pm 0.10$ & $\begin{array}{l}\uparrow=0.06 \\
P\end{array}$ & $M$ & $1.11 \pm 0.31$ & $1.03 \pm 0.39$ \\
\hline $\mathrm{F}$ & $0.73 \pm 0.12$ & $0.75 \pm 0.11$ & $\mathrm{~F}$ & $1.08 \pm 0.20$ & $0.96 \pm 0.35$ \\
\hline
\end{tabular}

(a) $w_{m 1}^{2}$ (weight or salience of the R-G axis of individual color space for observer $m$ ). (b) $w_{m 3}^{2}$ (salience of the Lightness axis) (c) $l_{m}^{2}$ (likelihood or goodness-of-fit between observer $m$ 's data and individual color space). And for completeness (d) $w_{m 2}^{2}$ (salience of the B-Y axis) for male (M) and female (F) nonsmokers and smokers. Arrows mark significant group differences. Dashed arrows mark suggestive group differences that do not meet the $P<0.05$ significance level. 
threshold for color differences to become perceptible was increased, as measured by increased errors when subjects arrange stimuli in hue sequence. Here we examined suprathreshold dissimilarities. Such data are less sensitive to loss of discrimination, but can detect changes in relative salience of color-space axes when analyzed with MDS. Instead of by sequencing errors, color deficit in a subject is signaled by dissimilarity judgments that (1) indicate that color displacements along one axis of color space are less salient relative to others (i.e. contribute less to overall dissimilarity); or (2) are incompatible with interstimulus distances in a spatial model (even a $\mathbf{X}_{m}$ optimally distorted for that subject). As noted in the Introduction, dissimilarities are possibly more responsive than discrimination judgements to global distortions of color space.

A previous study (Bimler et al., 2004) reported a group difference between males and females in the salience of the $R-G$ axis. Because our subjects included a disproportionate number of smoking males and nonsmoking females, any gender difference had the potential to create spurious results. Thus, male and female smokers and nonsmokers were compared separately. The key results are that smokers are impaired relative to nonsmokers. Among females, smokers placed less weight on red-green differences and more on lightness; among males, they were lower on overall performance. Clearly, this needs to be replicated with larger groups.

The polarity of any deficit is crucial; there are other ways to test it, for example, by systematically measuring thresholds along a range of directions in the color plane (Regan et al., 1994). Any damage to photoreceptors from smoking would more probably reduce blue-yellow salience (acquired tritanopia), because bluesensitive cones are relatively fragile (e.g. Hood \& Greenstein, 1988; Sperling, 1991). According to Köllner's law, the opposite finding suggests damage to the optic nerve. Some kind of impaired conduction accords with reports of increased reaction time to colored stimuli among smokers (Luria \& McKay, 1979). Combined with the aggravating factors of alcohol and malnutrition, smoking can bring about tobacco-alcohol amblyopia, a form of TXON (Krastel \& Moreland, 1991), with inhalation of CO and cyanide implicated in the etiology. It may be that the deficit encountered here is a subclinical form of the condition.

Chronic changes exist in smokers, rendering their darkadapted vision more vulnerable to $\mathrm{CO}$ exposure even without a recent cigarette (von Restorff \& Hebisch, 1988). However, the present study cannot distinguish chronic effects of smoking from immediate, short-term ones. Probably many of the smokers had smoked an hour to two prior to testing: though desirable, overnight abstinence was too much to request (they received only token payments).
Any future study should record the quantity smoked, to test earlier reports that the deleterious effects on color perception are dose dependent (Erb et al., 1999). Another possibly important variable is duration of smoking, although the effects of smoking are not necessarily progressive after the first year or two (Luria \& McKay, 1979).

\section{References}

Bimler, D.L., Kirkland, J. \& Jacobs, R. (2000). Colour-vision tests considered as a special case of multidimensional scaling. Color Research and Application 25, 160-169.

Bimler, D.L., Kirkland, J. \& Jameson, K.A. (2004). Quantifying variations in personal color spaces: Are there sex differences in color vision? Color Research and Application 29, 128-134.

Chang, J.J. \& CARroll, J.D. (1980). Three are not enough: An INDSCAL analysis suggesting that color space has seven $( \pm 1)$ dimensions. Color Research and Application 5, 193-206.

Erb, C., Nicaeus, T., Adler, M., Isensee, J., Zrenner, E. \& Thiel, H.-J. (1999). Colour vision disturbances in chronic smokers. Graefe's Archive of Clinical and Experimental Ophthalmology 237, 377-380.

FARnsworTh, D. (1943). The Farnsworth-Munsell 100-hue and dichotomous tests for color vision. Journal of the Optical Society of America 33, 568-578.

Fletcher, R. \& Voke, J. (1985). Defective Colour Vision: Fundamentals, Diagnosis and Management. Bristol, UK: Adam Hilger.

HeLm, C.R. (1964). Multidimensional ratio scaling analysis of perceived color relations. Journal of the Optical Society of America 54, 256-262.

Hood, D.C. \& Greenstein, V.C. (1988). Blue (S) cone pathway vulnerability: A test of a fragile receptor hypothesis. Applied Optics $\mathbf{2 7}$ 1025-1029.

Krastel, H. \& Moreland, J.D. (1991). Colour vision deficiencies in ophthalmic diseases. In Inherited and Acquired Colour Vision Deficiencies, ed. Foster, D.H., pp. 115-172. Basingstoke, UK: MacMillan.

Luria, S.M. \& McKAY, C.L. (1979). Visual processes of smokers and nonsmokers at different ages. Archives of Environmental Health 34, 449-454.

OFFENBACH, S.I. (1980). Children's perception of Munsell colors. Journal of Psychology 104, 43-51.

Regan, B.C., RefFin, J.P. \& Mollon, J.D. (1994). Luminance noise and the rapid determination of discrimination ellipses in colour deficiency. Vision Research 34, 1279-1299.

Shepard, R.N. \& CoOper, L.A. (1992). Representation of colors in the blind, color-blind, and normally sighted. Psychological Science 3, 97-104.

SPERLING, H.G. (1991). Vulnerability of the blue-sensitive mechanism. In Inherited and Acquired Colour Vision Deficiencies, ed. Foster, D.H., pp. 72-87. Basingstoke, UK: MacMillan.

TAKANE, Y. (1978). A maximum likelihood method for nonmetric multidimensional scaling: I. The case in which all empirical pairwise orderings are independent-Theory. Japanese Psychological Research 20, 7-17.

von Restorff, W. \& Hebisch, S. (1988). Dark adaptation of the eye during carbon monoxide exposure in smokers and nonsmokers. Aviation, Space, and Environmental Medicine 59, 928-931. 
Multidimensional scaling of D15 caps:

Color-vision defects among tobacco smokers?

Bimler, David L

2004

http://hdl.handle.net/10179/9667

22/04/2023 - Downloaded from MASSEY RESEARCH ONLINE 